\title{
Design of Automatic Integration Algorithm for Popular Science Microanimation Works in the Context of New Media
}

\author{
Xiaowen Shan (iD) and Hao Chen \\ Zhongbei College, Nanjing Normal University, Zhenjiang, Jiangsu 212300, China \\ Correspondence should be addressed to Xiaowen Shan; lingqing@njnu.edu.cn
}

Received 27 August 2021; Revised 1 October 2021; Accepted 9 October 2021; Published 21 October 2021

Academic Editor: Rahman Ali

Copyright (C) 2021 Xiaowen Shan and Hao Chen. This is an open access article distributed under the Creative Commons Attribution License, which permits unrestricted use, distribution, and reproduction in any medium, provided the original work is properly cited.

\begin{abstract}
When traditional methods integrate popular science microanimation works, the integration effect of the animation works is not good. In this paper, we propose an automatic integration algorithm of popular science microanimation works in the context of new media. The system first analyzes the characteristics of the new media context and gives the meaning of microanimation in the context of new media. It simplifies the edge folding of popular science microanimation integration and calculates the Facial Animation Parameter (FAP) value to realize the automatic integration of popular science microanimation works. We conducted a number of experiments using various size datasets to test the proposed system. We achieved an average integration accuracy of $96.3 \%$ with datasets of 500 to 3000 animation works, having the highest accuracy of $99 \%$ with a dataset of 500 animation works. On the other hand, the integration time of the animation works was recorded just 1.25 seconds with a dataset of 3000 animation works which is much lower than the existing work.
\end{abstract}

\section{Introduction}

The term "new media" was first used in 1967 when Godmark, director of CBS Technology Research Institute, first used "new media" in a commodity plan. By "new media," he means electronic video. In 1969, E. Rostow, chairman of the Sresidential Special Committee on American Communication Policy, also quoted the word "new media" in a report. Since then, the new media has attracted extensive attention and debate [1]. When new media was first proposed, it was actually endowed with the meaning of constant change. With the development of the times, technological innovation, and the development of media, it has been going through a process of pushing through the old and bringing forth the new. However, many basic problems about new media have not been solved. For example, the authoritative definition of new media has not been established, and the research object is still lack of specific division. The author believes that to solve these problems, the most fundamental thing is to make a definition of new media [2]. Portals and microblogs call themselves new media, Internet and TV call themselves new media, and paper media has also been revised to dress up as new media. This chaotic situation is in line with the prediction of American communication scholar Schramm: "the basic nature of human communication will not change, but the social system of communication itself is likely to be different from that in previous periods" [3]. To define new media from the perspective of communication, "new media" should have the communication characteristics that traditional media do not have. Then, different modes of communication should be used to distinguish standards. If the media initially carried out interpersonal communication through the "point-to-point" way of language and sound, the emergence of paper media has transitioned to "point-topoint" mass communication. Finally, the emergence of new media has brought about the change of new communication mode, that is, "everyone to everyone communication" [4]. The most important characteristic of new media is interactivity, and the disseminator is also the receiver. In this way, new media cannot be simply divided from technology and terminals. For example, mobile microblog is a new media, and mobile newspaper is still among the traditional media 
although it is equipped with the electronic terminal of mobile phone. At the same time, it is precise because of this interaction of new media that it combines producers and consumers more closely than the unidirectional nature of traditional media [5].

Therefore, new media should be based on computer technology, communication technology, digital broadcasting, and other mechanisms to realize personalized, differentiated, and interactive communication through Internet, digital radio and television network, satellite, and other channels. In this new media era, there are two keywords, namely, UGC (user-generated content) and CGM (consumer-generated media) [6]. These two keywords also highlight the two major trends of the improvement of audience initiative and media professional segmentation in the new media era.

There are a number of relevant studies that focused on the use of integration algorithms for various purposes $[6,7]$. However, their focus areas are different. Literature [6] talks about the integration of work-life boundaries of female nurses in hospitals while literature [7] focuses on "work-tononwork" of workers in different organizations. Moreover, the systems they proposed have lower integration accuracies that need to be enhanced, and their systems are not as time efficient as they should be.

To address these limitations and to present a novel work in the area of microanimations, we propose an automatic integration system of popular science microanimation works in the context of new media. The system reads the microanimation model, loads the Face Animation Parameters, and maps them with Face Definition Parameters (FDPs) to calculate the scope of FDP influence. It draws a new model of the microanimation and displays the result of its composition.

Key contributions of the study include (1) the adoption of the Candide-3 model [8] in the educational microanimation, (2) the introduction and integration of new media contents in the educational environment, and (3) automatic extraction of the users preferences, reviews, and comments from the microanimation at run time and their integration to the system to improve the learning aspect of the students via the updated microanimations.

The rest of the paper is organized as follows. Section 2 talks about microanimations and its characteristics. In Section 3, we discuss the automatic integration algorithm. In Section 4, we discuss our proposed system in detail. Section 5 talks about the experimental setup and results, and Section 6 is the conclusion of our study.

\section{Analysis of Microanimation Work in the Context of New Media}

Microanimations are small but powerful tools to make data more understandable, make the UI design better, enhance the user experience, and visualize the processes or results in a better way. In this section, we will discuss the characteristics and some applications of new media.

\subsection{Characteristics of New Media Context.}

(1) Lower Threshold for Communicators. Traditional media such as newspaper, radio, and television always has a relatively fixed role division of communicator and audience. However, the arrival of the new media era also announces the advent of an era in which everyone has a microphone. No matter where you are in the world, chances are you will be in the spotlight.

(2) The mode of transmission presents a cracking type. Information transmission in the new media era can be carried out simultaneously by many terminals. After receiving the information, the audience can immediately forward and attach their own comments. In just a few tens of seconds, the transformation from the information receiver to the publisher is completed [7]. This kind of cracking mode of transmission is most likely to produce the "aggregation outbreak" effect; an originally insignificant message, through this kind of cracking forwarding, is likely to become the topic of public discussion immediately.

(3) The forms of expression become rich and diversified. The communication mode of new media is the link connecting information [9]. The development of new media has brought a big explosion of information. A message can have multiple forms of expression such as video, audio, and text, and the audience can also easily search for relevant information through links or search engines [10]. Various forms of expression enable new media to combine the advantages of various traditional media in one. Video and audio messages are great for creating atmosphere or a strong sense of presence. And text is more conducive to in-depth reading and thought-provoking.

(4) The audience are becoming more and more fragmented. With the development of new media, the agenda-setting function of traditional media has been eliminated, and audience have more and more options. Moreover, information publishers can timely capture the preferences of audience through their click-through rates and feedback [11]. According to the preferences of the audience, the media subdivides many channels or sections such as entertainment, music, automobile, and drama to meet the needs of the audience.

(5) The information content is made briefer. The development of new media has also led to the arrival of the era of short reading. Microblog and microfilm are becoming more and more popular [12]. As the pace and intensity of work increase, people's time becomes more and more fragmented. Large chunks of reading time are being cut up into smaller chunks, leaving less and less time for deep reading. In order to adapt to this trend, the content of media dissemination is becoming shorter and shorter. 
2.2. Educational "Microanimation" in the Context of New Media. New media is a creative platform for educational "microanimation," and it can also carry out intuitive evaluation of animation works. The source of creativity comes from life, and the outburst of creativity often comes from communication. The biggest difference between new media and traditional media is that new media has stronger instant interactivity. This interaction brings more creative inspiration to educational "microanimation." Through instant interaction and communication, netizens show the most life-like things [13]. All the Internet languages are produced by netizens, "micro-animation" became popular, and its role is to conduct more research on the audience's mentality. New media offers a good opportunity to educate "microanimation" makers because new media platforms are more open than traditional media and educational "microanimation." At the same time, when traditional media broadcasts educational animation, the popularity can only be calculated by the data of audience rating. It takes a lot of statistical work to know who the main audience are, why they like them, and which characters they like. However, on the new media platform, the comments of the audience are easy to obtain, and the preferences of the audience can also be monitored in real time through statistics, which is of great benefit to some animations with relatively long broadcast cycle [14]. "Microanimation" can learn what the audience like as it plays and make adjustments to the episodes that do not play.

On the new media platform, it is easier to produce the "burst of aggregation" effect. In traditional media, the broadcast of animation has to go through a heavy review, focusing on its social and educational significance. The plot design of many animations is old-fashioned and not close to the current real life, so the audience do not like it, and the educational significance is naturally difficult to achieve [15]. However, in the new media platform, the design of "microanimation" can use more network language, and the timeliness is also greatly strengthened. The era of new media is an era in which the taste of wine is also afraid of the deep lanes. There are various marketing and promotion methods and various publicity channels and forms. If the content producers can make good use of various resources, they can often play a very good role.

New media is a high-quality e-commerce platform with both communication and operation functions. For traditional media, the media mainly plays the role of communication, and a lot of operation and distribution work is carried out offline. The new media platform is not only a good communication platform but also a marketing platform. Therefore, "microanimation" public relations publicity is also very important. We have already entered an era of eyeball economy, the production, distribution, and operation of the three carriages to promote the overall development of educational animation. The three complement each other and are indispensable. To promote the education of "microanimation" on new media, it is necessary to be familiar with the rules of new media communication and operation. Instead of taking a passive pr approach like the traditional one, we should actively promote it. Compared with offline promotion, online promotion is easy to produce small investment and big profit phenomenon. The release of educational "microanimation" should study the distribution rules of new media and actively participate in the investment so as to take the initiative in this era of scarcity of eyeballs.

\section{Automatic Integration Algorithm}

In this section, we will discuss the integration algorithm and its use for automatic integration of science microanimation works in the new media context.

\subsection{Loop Subdivision Optimization of Popular Science} Microanimation Works. Because Candide-3 model [8] structure is simple and the contained data volume is small, the Candide- 3 model to build the facial animation data is very simple. However, only 183 triangular meshes showing facial animation as truth is not enough. In conclusion, this paper adopts the segmentation algorithm to optimize the model and then makes the model in facial animation. Loop subdivision is a very simple triangular mesh-oriented optimization method proposed by Charles loop from the University of Utah. The loop segmentation model puts a triangle according to certain rules into four triangles, equivalent to the triangular mesh of each. The loop subdivision process on a triangular mesh is shown in Figure 1.

As shown in Figure 1, in the loop subdivision process of a triangular mesh, new vertices $E_{1}, E_{2}$, and $E_{3}$ inserted by each edge of the original mesh are called class I control points and updated vertices $V_{1}^{\prime}, V_{2}^{\prime}$, and $V_{3}^{\prime}$ corresponding to original mesh vertices $V_{1}, V_{2}$, and $V_{3}$ are called class II control points. The calculation rules of class I control points and class II control points in the loop subdivision algorithm are introduced as follows. Calculation template of class I control point of loop subdivision is shown in Figure 2.

3.1.1. Class I Control Points. As shown in Figure 2(a), when side $\left(V_{0}, V_{1}\right)$ is a nonboundary side, its two adjacent triangles are $\Delta\left(V_{0}, V_{1}, V_{2}\right)$ and $\Delta\left(V_{0}, V_{1}, V_{3}\right)$, respectively, and then the coordinates of class I control point $V_{E}$ can be calculated as follows:

$$
V_{E}=\frac{3}{8}\left(V_{0}+V\right)_{1}+\frac{1}{8}\left(V_{2}+V_{3}\right) .
$$

As shown in Figure 2(b), when edge $\left(V_{0}, V_{1}\right)$ is the boundary edge, the coordinate of class I control point factory $V_{E}$ inserted on boundary edge $\left(V_{0}, V_{1}\right)$ can be calculated by the following equation:

$$
V_{E}=\frac{1}{2}\left(V_{0}+V_{1}\right)
$$

3.1.2. Class II Control Points. Calculation template of loop subdivision of class II control points is shown in Figure 3.

As shown in Figure 3, if there are $n$ edges connected to vertex $V$ and the other endpoints of all edges where vertex $V$ is located are $V_{0}, V_{1}, V_{2}, \ldots, V_{n-1}$, respectively, then the coordinates of control point $V^{\prime}$ of class $n$ generated by updating vertex $V$ can be calculated by the following equation: 


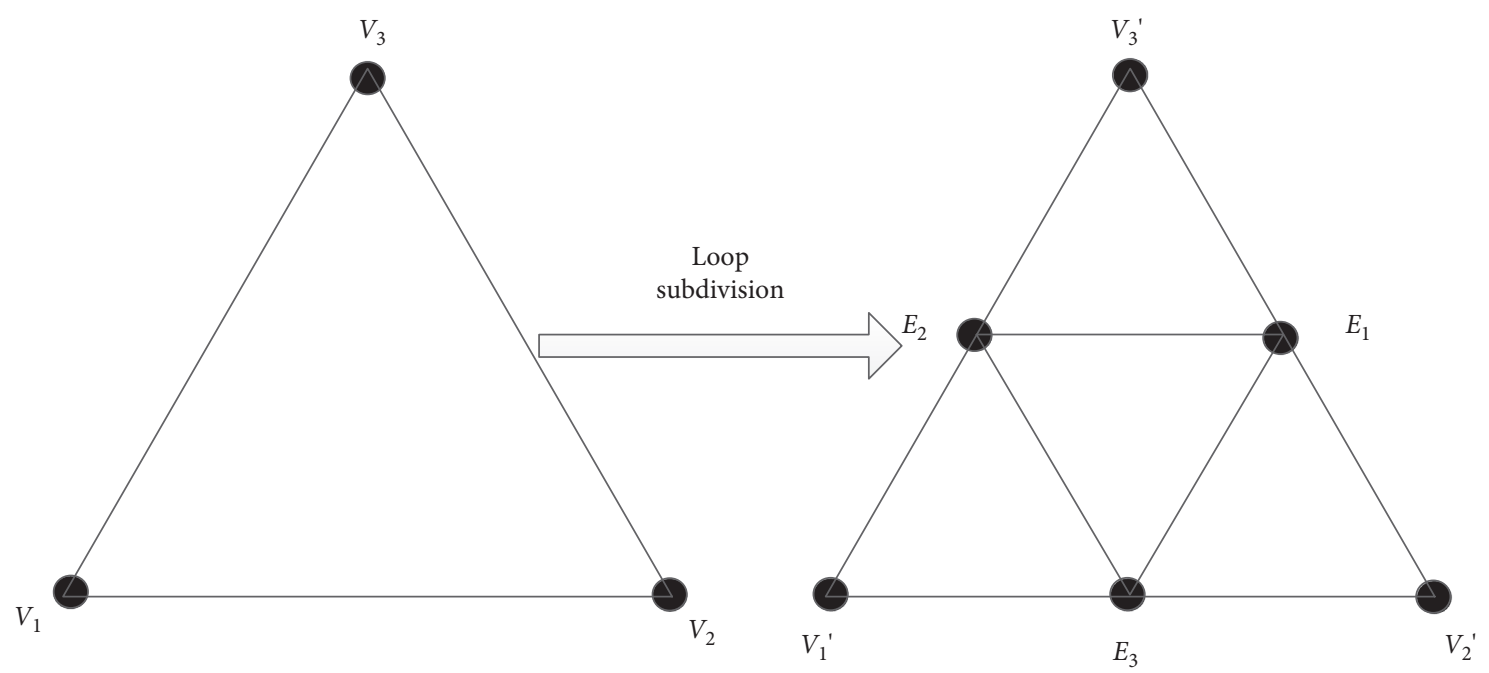

FIGURE 1: Loop subdivision process on a triangular mesh.

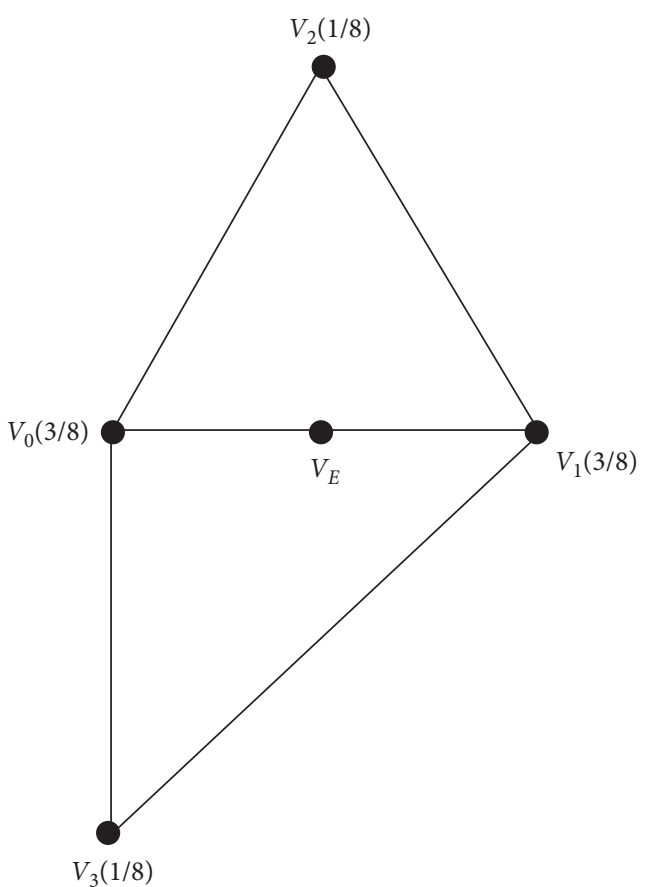

(a)

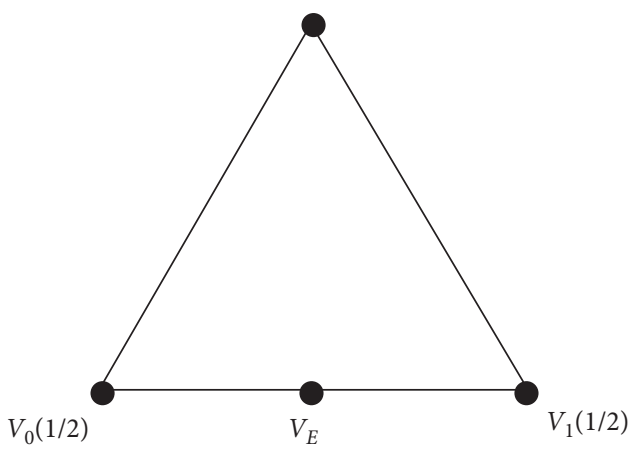

(b)

FIgURE 2: (a) A calculation template for class I control points on nonboundary edges. (b) A calculation template for class I control points on the boundary.

$$
V^{\prime}=\left(1-n \beta_{n}\right) V+\beta_{n} \sum_{i=0}^{n-1} V_{i}
$$

Among them, $\beta_{n}=(1 / n)[(5 / 8)-((3 / 8)+(1 / 4) \cos$ $\left.(2 \pi / n))^{2}\right]$.

3.2. Simplified Edge Folding Algorithm of Popular Science Microanimation Integration. In order to simplify the generation of the model in this paper, the loop subdivision method is used [16] to calculate the location of the new vertex $V_{\text {new }}$.
Under the same simplified rate, in order to keep the details of the 3D face model characteristics, such as facial features and contour features, this article defines the error measure based on the vertex weighted letter $D_{E}\left(, V_{\text {new }}\right.$, Cur $)$ where $E$ is the folded edge $\left(V_{i}, V_{j}\right)$, which is $E=\left(V_{i}, V_{j}\right)$. Considering the high requirement of triangular mesh quality for deformation control and rendering of the $3 \mathrm{D}$ virtual face model, this paper defines the triangular mesh shape attitude Tri_shape $\left(V_{i}, V_{j}\right)$ to represent the triangular mesh quality [17].

The error measure function $D_{E}\left(, V_{\text {new }}\right.$, Cur $)$ and triangular mesh attitude Tri_shape $\left(V_{i}, V_{j}\right)$ form a double- 


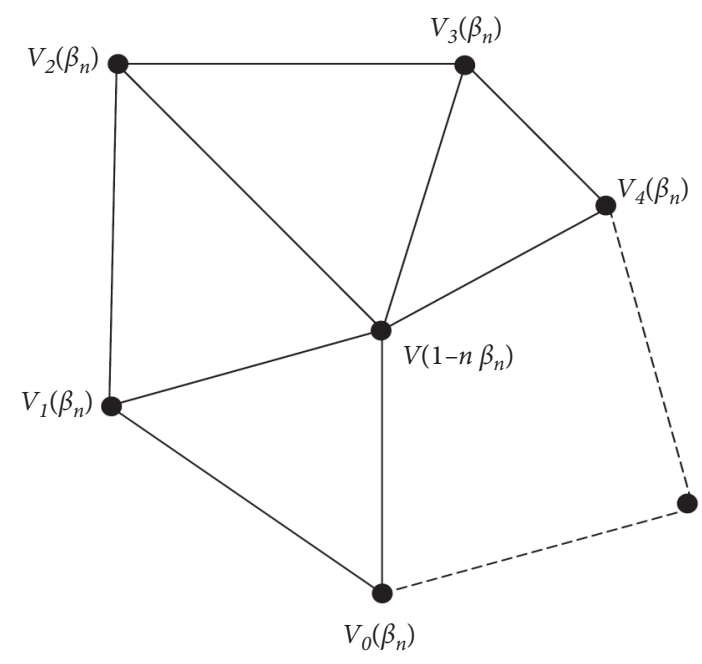

FIGURE 3: Calculation template of loop subdivision of class II control points.

constraint mechanism to control the edge folding order together, which ensures the preservation of model details and the generation of the high-quality simplified model. The double-constraint mechanism is measured by the doubleconstraint error measure function $\operatorname{Metric}\left(V_{i}, V_{j}\right)$, as shown in the following equation:

$$
\operatorname{metric}\left(V_{i}, V_{j}\right)=D_{E}\left(, V_{\text {new }}, \text { Cur }\right) \times \text { Tri_shape }\left(V_{i}, V_{j}\right) \text {. }
$$

The value of the double-constraint error measure function is taken as the edge folding cost. The queue generated by sorting the edges from small to large according to the folding cost is called the double-constraint folding sequence. In the queue, the edge $\left(V_{i}, V_{j}\right)$ that satisfies the minimum value of the double-constrained error measure function in equation (4) is called the edge to be folded.

\section{Automatic Integration Scheme of Popular Science Microanimation Works}

According to the working principle of the integration of popular science microanimation works, this section designs the integration scheme of popular science microanimation works. The steps are as follows.

(1) After reading the face model, according to the definition of each parameter in the MPEG-4 standard, FDP is mapped and FAPU is calculated, which is equivalent to the control point of the mapping model. As shown in Figure 4, the animation principle based on the MPEG- 4 standard is that FAP drives FDP, which affects the movement of the surrounding vertices. So, mapping control points are the basis for implementing the next steps.

(2) According to the control points of the FDP mapping model, the influence range of FDP is determined on the face model.
(3) Loading FAP, facial expression animation based on the MPEG- 4 standard is driven by FAP, equivalent to FAP which determines the composite result of facial model expression. FAP is independent only related to the expression and has nothing to do with the model, so this step can be done simultaneously with mapping FDP and calculating FAPU.

(4) Looking for face animation definition table FDT, each FAP has a face animation definition table corresponding to it. Using the FDT, the new grid vertex coordinates can be computed, so FDT is the key to affect the face animation.

(5) According to the size of the FAP value, the new coordinates of the mesh vertices of the FDP influence range are calculated.

(6) Based on the new coordinates of the model grid, the model is redrawn to show the emojis corresponding to the loaded FAP.

To sum up, the flow chart of the integration scheme of popular science microanimation works designed in this paper is shown in Figure 5.

\section{Calculation of Face Animation Parameter (FAP) Value}

In the facial expression synthesis process based on the MPEG-4 standard, the change of facial expression is driven by FAP, and the FAP value determines the intensity of facial expression. If you know the value of a FAP, then you can synthesize expressions that correspond to the magnitude of the value. Conversely, if you want to know the FAP value of a particular expression, you need to calculate it by the coordinates of the face model before and after deformation. The specific method of calculation is an inverse operation method. This process is necessary for this topic because this topic needs to synthesize 6 basic facial expressions, and a set of FAP is needed to drive the submodel to synthesize these 6 expressions. The size of FAP value is calculated according to the coordinates before and after the deformation by making the mother model generate the corresponding deformations of 6 basic facial expressions. The process is the construction of face specific expression animation data. The rest of this section will describe in detail the calculation method of face animation parameter FAP value.

When the model expression is neutral, set a FAP FDP mapping on the model of the feature point coordinates to $V(x, y, z)$, synthetic model deformation after an animation, the coordinates of the feature points to $V^{\prime}\left(x^{\prime}, y^{\prime}, z^{\prime}\right)$ if the FAP definition in the FDT field of the table is divided into one segment, the motion factor is $(a, b, c)$, and the following formula is an animation of the FAP value displayed by type (5):

$$
\left(\begin{array}{l}
x^{\prime} \\
y^{\prime} \\
z^{\prime}
\end{array}\right)=\mathrm{FAPU} \times \mathrm{FAP} \times\left(\begin{array}{l}
a \\
b \\
c
\end{array}\right)+\left(\begin{array}{l}
x \\
y \\
z
\end{array}\right) .
$$




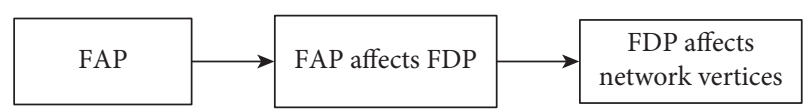

FIGURE 4: Schematic diagram of integration principle of popular science microanimation works based on the MPEG-4 standard.

By rearranging equation (5), the following equation is obtained:

$$
\left(\begin{array}{c}
\mathrm{FAPU} \times \mathrm{FAP} \times a \\
\mathrm{FAPU} \times \mathrm{FAP} \times b \\
\mathrm{FAPU} \times \mathrm{FAP} \times c
\end{array}\right)=\left(\begin{array}{c}
x^{\prime}-x \\
y^{\prime}-y \\
z^{\prime}-z
\end{array}\right)
$$

Then, the value of FAP can be obtained by equation (7) or $(8)$ or $(9)$.

$$
\begin{aligned}
& \mathrm{FAP}=\frac{\left(x^{\prime}-x\right) / a}{\mathrm{FAPU}}, \\
& \mathrm{FAP}=\frac{\left(y^{\prime}-y\right) / b}{\mathrm{FAPU}}, \\
& \mathrm{FAP}=\frac{\left(z^{\prime}-z\right) / c}{\mathrm{FAPU}} .
\end{aligned}
$$

At this time, the integration algorithm of microanimation works is realized.

\section{Experiments}

In this section, we are going to discuss the experimental setup of the proposed system and discuss the experimental results and compare them to the existing work.

6.1. Experimental Setup. In this paper, the development environment used has the following specifications:

(i) CPU: Intel Core, $2.0 \mathrm{GHz}$

(ii) Memory: 2.0 GB

(iii) Operating system: Windows 7

(iv) IDE: Visual Studio 2010

(v) Programming language: $\mathrm{C}++$

We use MFC (Microsoft Foundation Class) to establish OpenGL 3D drawing framework. The 3D facial expression synthesis system designed is based on the MPEG-4 standard. The animation works automatically integrate the parameter FAP to control the virtual character's facial expression animation.

6.2. Animation Works Integration Accuracy. In order to verify the integration effect of the method in this paper on the automatic integration algorithm of popular science microanimation works in the context of new media, literature [6] method, literature [7] method, and the animation work integration algorithm of the method in this paper are adopted, and the experimental results are shown in Table 1. Literature [6] is an extensive study of female nurses working

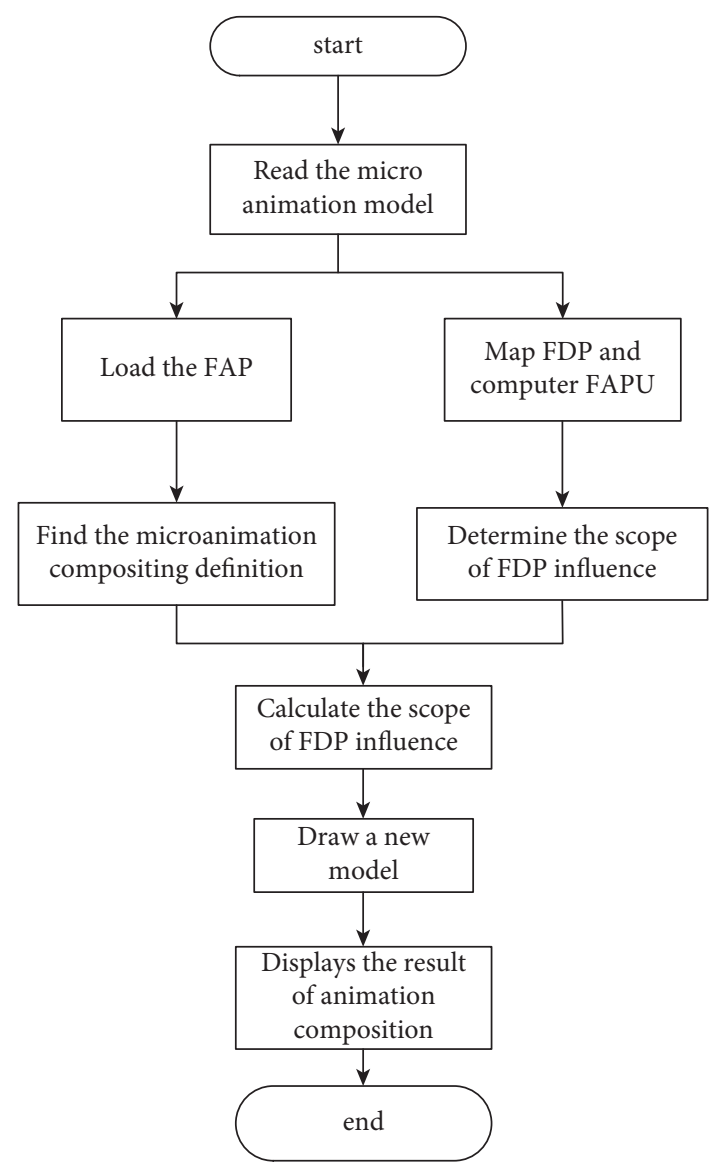

FIGURE 5: Integration flow chart of popular science microanimation works.

in hospitals. The authors have studied and discussed a number of factors that affect the work-life boundaries of the nurses. They collected a dataset of 724 nurses to conduct their experiments and present the information that how boundary integration and life satisfaction are closely related. Similarly, literature [7] is about "work-to-nonwork" integration of employees by using RAA (Reasoned Action Approach) framework. We propose an automatic integration algorithm of popular science microanimation works in the context of new media. All these studies use integration algorithms in their domains. To the best of our knowledge, these studies are the closest related studies to our work and our work is the first to focus on microanimation works.

Analysis of Table 1 shows that the integration accuracy of animation works is different under different methods. When the number of animation works is 100 , the integration accuracy of the method of literature [6] is $78 \%$, the integration accuracy of the method of literature [7] is $74 \%$, and the integration accuracy of animation works by our proposed method is $93 \%$. When the number of animation works is 500 , the integration accuracy of the method of literature [6] is $69 \%$, the integration accuracy of the method of literature [7] is $57 \%$, and the integration accuracy of animation works by the method of this paper is $99 \%$. It is obvious from Table 1 that accuracy of animation integration of this method is much higher than that of traditional methods. 
TABLE 1: Integration accuracy of animation works with different methods.

\begin{tabular}{|c|c|c|c|}
\hline \multirow{2}{*}{ Number of animation works/piece } & \multicolumn{3}{|c|}{ Integration accuracy of animation works with different methods (\%) } \\
\hline & Literature [6] methods & Literature [7] methods & Our method \\
\hline 100 & 78 & 74 & 93 \\
\hline 200 & 76 & 79 & 96 \\
\hline 300 & 72 & 76 & 98 \\
\hline 400 & 68 & 63 & 95 \\
\hline 500 & 69 & 57 & 99 \\
\hline 600 & 71 & 59 & 97 \\
\hline
\end{tabular}

TABLE 2: Time for automatic integration of popular science microanimation works.

\begin{tabular}{lcc}
\hline \multirow{2}{*}{$\begin{array}{l}\text { Number of animation works/piece } \\
\text { Literature [6] methods }\end{array}$} & \multicolumn{2}{c}{ Automatic integration of popular science microanimation works (s) } \\
Literature [7] methods
\end{tabular}

6.3. Time for Automatic Integration of Animation Works. In order to verify the automatic integration efficiency of the method for popular science microanimation works in the context of new media, the automatic integration time of animation works using the methods of literature [6], literature [7] and this paper is compared, and the experimental results are shown in Table 2.

It is obvious from the data of Table 2 that our proposed system outperforms the traditional approach in terms of time taken too. For a dataset size of 500 samples, literature [6] method required 39 seconds, literature [7] method took 38 seconds while our proposed system took only $0.16 \mathrm{sec}-$ onds. Similarly for a large size dataset of 3000 samples, literature [6] method took 100 seconds, literature [7] method took 124 seconds while our proposed method elapsed only 1.25 seconds. The rationales behind the low time complexity of the proposed method as compared with the competing methods are that the proposed method deals over microanimation designed in UI/UX animation and motion design tools while the competitive methods focus the new media contents which are designed using the new media technologies, comprising virtual art, computer graphics, and computer animation.

\section{Conclusion}

This paper proposes an automatic integration algorithm of Kopp microanimation works in the context of new media. The characteristics of new media context are analyzed, and loop subdivision and optimization of popular science microanimation works are carried out to simplify the edge folding of popular science microanimation integration. An automatic integration scheme of popular science microanimation works is given, the edge folding of the microanimation integration is simplified, and the FAP value is calculated to realize the automatic integration of the microanimation works. The proposed system achieves the integration accuracy of $99 \%$ with a dataset of 500 samples. On the other hand, the integration time of microanimation works is just 1.25 seconds for a dataset of 300 samples indicating the time efficiency of the proposed system.

\section{Data Availability}

The data used to support the findings of this study are available from the corresponding author upon request.

\section{Conflicts of Interest}

The authors declare that there are no conflicts of interest.

\section{Acknowledgments}

This study was supported by School Level Project of Nanjing Normal University Zhongbei College: "Research on the design and production of popular science microanimation in the context of new media" (Grant no. 2020yky007).

\section{References}

[1] D. Tait Shanafelt, P. Colin, C. Sinsky et al., "Changes in burnout and satisfaction with work-life integration in physicians and the general US working population between 2011 and 2017," Mayo Clinic Proceedings, vol. 12, no. 25, pp. 88-98, 2019.

[2] N. Calvo, M. Rey-Garcia, and V. Mato-Santiso, "Survival dynamic of strategic alliances for work integration," in Proceedings of the ACEDEGlobalization under complexity and uncertainty environments, Coruña, Spain, November 2019.

[3] A. Signoretti and S. Sacchetti, "Lean HRM practices in work integration social enterprises: moving towards social lean production. Evidence from Italian case studies*," Annals of Public and Cooperative Economics, vol. 76, no. 32, pp. 87-99, 2020. 
[4] L. V. Pavo, C. B. Miranda, J. A. Caballero, A. S. S. R. Mauro, and B. B. C. Caliane, "Multiperiod work and heat integration," Energy Conversion and Management, vol. 227, no. 32, pp. 113-187, 2021.

[5] L. N. Dyrbye, C. P. West, M. Halasy, D. J. O'Laughlin, D. Satele, and T. Shanafelt, "Burnout and satisfaction with work-life integration among PAs relative to other workers," Journal of the American Academy of Physician Assistants, vol. 33, no. 5, pp. 35-44, 2020.

[6] M. Yasir, A. Majid, M. Yasir, and N. Khan, "Boundary integration, work/family enrichment and life satisfaction among female nursing staff," Management Research Review, vol. 42, no. 6, pp. 740-759, 2019.

[7] E. A. Cavallo, A. E. Fernández, and M. Matías, "Making international financial integration work for low-saving countries," IDB Publications (Working Papers), vol. 65, no. 43, pp. 99-124, 2020.

[8] J. Ahlberg, "Candide-3-an updated parameterised face," LiTHISY-R-2326, Department of Electrical Engineering, Linköping University, Linkoping, Sweden, 2001.

[9] E. Palm, C. Seubert, and J. Glaser, "Understanding employee motivation for work-to-nonwork integration behavior: a reasoned action approach," Journal of Business and Psychology, vol. 21, no. 3, pp. 1-14, 2019.

[10] J. Andrew, E. Aurora, C. Perri et al., "The health and wellbeing impacts of a work integration social enterprise from a systems perspective," Health Promotion International, vol. 65, no. 32, pp. 88-107, 2021.

[11] A. Knigs, T. Radtke, J. Braun, X. Chen, H. Dressel, and C. Benden, "A bumpy road: work integration of people with cystic fibrosis after lung transplantation," The Journal of Heart and Lung Transplantation, vol. 39, no. 4, pp. 209-225, 2020.

[12] J. Sforzi and L. A. Colombo, "New opportunities for work integration in rural areas: the 'social flavour' of craft beer in Italy,” Sustainability, vol. 12, no. 16, pp. 6351-6379, 2020.

[13] K. N. Ohei and R. Brink, "Employability distress: factors affecting ICT graduates' employability and work integration in the workspace," Research in World Economy, vol. 12, no. 1, pp. 138-149, 2021.

[14] B. Dufour, F. Petrella, and N. Richez-Battesti, "Understanding social impact assessment through public value theory: a comparative analysis on work integration social enterprises (WISEs) in France and Denmark," Working Papers, vol. 41, no. 27, pp. 112-138, 2020.

[15] F. Majetić, M. Makarovič, D. Šimleša, and T. Golob, "Performance of work integration social enterprises in Croatia, Slovenia, and Italian regions of Lombardy and Trentino," Economics \& Sociology, vol. 12, no. 1, pp. 286-301, 2019.

[16] H. Li and S. Kim, "Mesh simplification algorithms for rendering performance," International Journal of Engineering Research and Technology, vol. 13, no. 6, pp. 1110-1119, 2020.

[17] C. P. Agustin, "Work integration on the family life of professional working mothers," International Journal of Psychosocial Rehabilitation, vol. 24, no. 3, pp. 2290-2303, 2020. 